Supporting Information for

\title{
Kinetic Analysis of Competitive Electrocatalytic Pathways: New Insights into Hydrogen Production with Nickel Electrocatalysts
}

\author{
Eric S. Wiedner,* Houston J. S. Brown, and Monte L. Helm \\ Center for Molecular Electrocatalysis, Pacific Northwest National Laboratory, \\ P.O. Box 999, K2-57, Richland, Washington 99352, USA \\ *E-mail: eric.wiedner@pnnl.gov
}

Table of Contents

Page

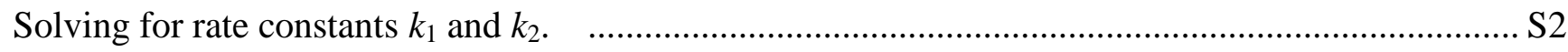

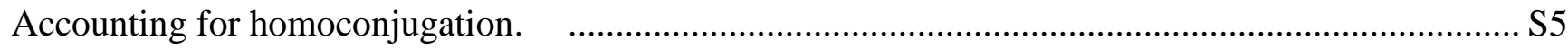

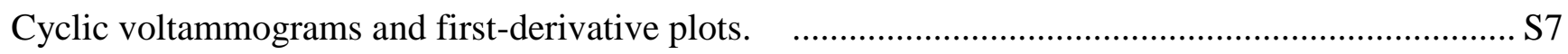

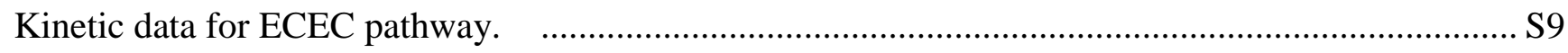

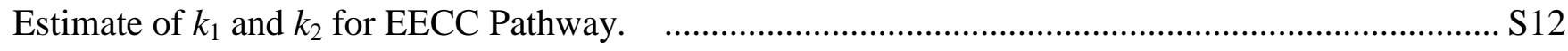

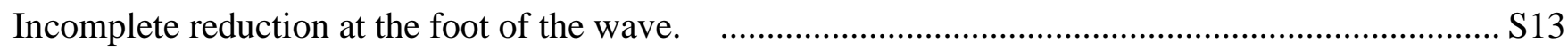

FOWA of 1 with unbuffered $\operatorname{DMF}(\mathrm{H})^{+}$. 


\section{Solving for Rate Constants $k_{1}$ and $k_{2}$}

For both the ECEC and EECC mechanisms, $k_{\mathrm{obs}}$ is defined by:

$\sqrt{k_{o b s}}=\frac{\sqrt{k_{1}}}{1+\lambda}$

In the kinetic regime where $k_{1}>k_{2}$, both $k_{\text {obs }}$ and $\lambda$ can be measured from experimental catalytic waves (equations 3 and 5 in the main text), and $k_{1}$ is readily solved in terms of $k_{\mathrm{obs}}$ and $\lambda$ :

$k_{o b s}=\frac{k_{1}}{(1+\lambda)^{2}}$

$\therefore k_{1}=k_{o b s}(1+\lambda)^{2}$

$\lambda$ is mechanism-dependent and is defined by $k_{1}$ and $k_{2}$. Since $k_{1}$ can be determined as shown above, $k_{2}$ can be determined from $k_{1}$ and $\lambda$ :

$\underline{\text { ECEC Mechanism }}$

$\lambda=\frac{\sqrt{k_{1}}}{\sqrt{k_{2}}}$

$\therefore k_{2}=\frac{k_{1}}{\lambda^{2}}$

$\underline{\text { EECC Mechanism }}$

$\lambda=\frac{\sqrt{k_{1}}}{\sqrt{k_{2}}\left(1+\frac{\sqrt{k_{2}}}{\sqrt{k_{1}}}\right)}$

$\lambda \sqrt{k_{2}}\left(1+\frac{\sqrt{k_{2}}}{\sqrt{k_{1}}}\right)=\sqrt{k_{1}}$

Multiply both sides by $\sqrt{k_{1}}$ and collect similar terms:

$\lambda \sqrt{k_{2}}\left(\sqrt{k_{1}}+\sqrt{k_{2}}\right)=k_{1}$

$\lambda \sqrt{k_{1}} \sqrt{k_{2}}+\lambda k_{2}=k_{1}$

$k_{1}-\lambda k_{2}=\lambda \sqrt{k_{1}} \sqrt{k_{2}}$ 
Square both sides and collect terms in the form of a quadratic equation of $k_{2}$ :

$$
\begin{aligned}
& k_{1}^{2}-2 \lambda k_{1} k_{2}+\lambda^{2} k_{2}^{2}=\lambda^{2} k_{1} k_{2} \\
& \lambda^{2} k_{2}^{2}-\left(2 \lambda k_{1}+\lambda^{2} k_{1}\right) k_{2}+k_{1}^{2}=0
\end{aligned}
$$

Apply the quadratic formula and simplify terms:

$$
\begin{aligned}
& k_{2}=\frac{-\left[-\left(2 \lambda k_{1}+\lambda^{2} k_{1}\right)\right] \pm \sqrt{\left[-\left(2 \lambda k_{1}+\lambda^{2} k_{1}\right)\right]^{2}-4 \lambda^{2} k_{1}^{2}}}{2 \lambda^{2}} \\
& k_{2}=\frac{\left(2 \lambda k_{1}+\lambda^{2} k_{1}\right) \pm \sqrt{\left(4 \lambda^{3}+\lambda^{4}\right) k_{1}^{2}}}{2 \lambda^{2}} \\
& k_{2}=\frac{\left(2 \lambda+\lambda^{2}\right) k_{1} \pm\left(\sqrt{4 \lambda^{3}+\lambda^{4}}\right) k_{1}}{2 \lambda^{2}} \\
& k_{2}=\frac{2 \lambda+\lambda^{2} \pm \sqrt{4 \lambda^{3}+\lambda^{4}}}{2 \lambda^{2}} k_{1} \\
& k_{2}=\left(\frac{2 \lambda}{2 \lambda^{2}}+\frac{\lambda^{2}}{2 \lambda^{2}} \pm \frac{\sqrt{4 \lambda^{3}+\lambda^{4}}}{2 \sqrt{\lambda^{4}}}\right) k_{1} \\
& \therefore k_{2}=\left(\frac{1}{\lambda}+\frac{1}{2} \pm \frac{1}{2} \sqrt{\frac{4+\lambda}{\lambda}}\right) k_{1}=C k_{1}
\end{aligned}
$$

This gives two root values for $k_{2}$, only one of which can be correct. For the case where $k_{1} \geq k_{2}$, then by definition $\lambda \geq 0.5$ and the complex coefficient $(C)$ in the equation above must satisfy the conditions of $1 \geq C>0$. Figure $S 1$ below shows a plot of $C$ versus $\log (\lambda)$ for the coefficients of the positive and negative roots, $C(+)$ and $C(-)$. As $\lambda$ is increased from $0.5, C(+)$ decreases from an initial value of 4 and approaches (but does not reach) a value of 1 at large values of $\lambda$. This does not satisfy the conditions outlined above, so $C(+)$ cannot be used to obtain the correct value of $k_{2}$. In contrast, $C(-)$ has a value of 1 at $\lambda=0.5$, and increasing $\lambda$ causes $C(-)$ to decrease and approach (but not reach) a value of 0 . Therefore $C(-)$ satisfies the conditions outlined above and can be used to measure the correct value of $k_{2}$ :

$k_{2}=\left(\frac{1}{\lambda}+\frac{1}{2}-\frac{1}{2} \sqrt{\frac{4+\lambda}{\lambda}}\right) k_{1}$ 


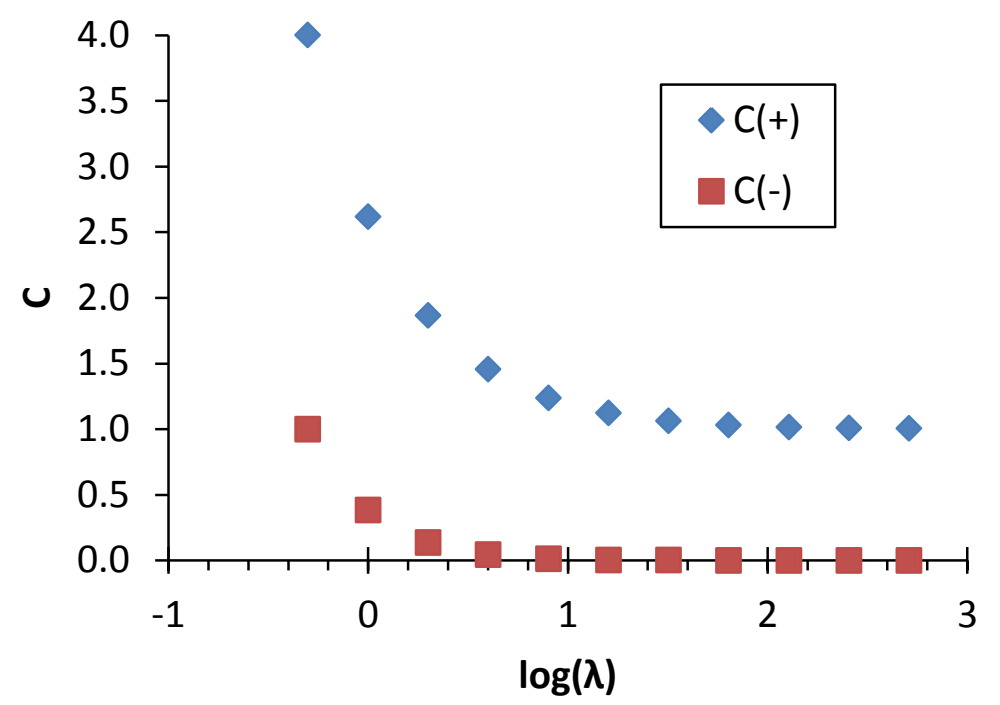

Figure S1. Plot of $C$ versus $\log (\lambda)$ for an EECC mechanism with $k_{1} \geq k_{2}$, where $C(+/-)=\left(\frac{1}{\lambda}+\frac{1}{2} \pm \frac{1}{2} \sqrt{\frac{4+\lambda}{\lambda}}\right)$ 


\section{Accounting for Homoconjugation}

General Equations:

$\mathrm{BH}^{+}+\mathrm{B} \rightleftharpoons \mathrm{BHB}^{+} \quad K_{\mathrm{f}}=\frac{\left[\mathrm{BHB}^{+}\right]}{\left[\mathrm{BH}^{+}\right][\mathrm{B}]}$

$\left[\mathrm{BH}^{+}\right]_{\mathrm{o}}+[\mathrm{B}]_{\mathrm{o}}=\left[\mathrm{BH}^{+}\right]+[\mathrm{B}]+2\left[\mathrm{BHB}^{+}\right]$

Determine $\left[\mathrm{BH}^{+}\right]_{\mathrm{EQ}}$ for initial concentrations $\left[\mathrm{BH}^{+}\right]_{\mathrm{o}}$ and $[\mathrm{B}]_{\mathrm{o}}$ :

$\left[\mathrm{BH}^{+}\right]=x ;\left[\mathrm{BHB}^{+}\right]=\left[\mathrm{BH}^{+}\right]_{\mathrm{o}}-x ;[\mathrm{B}]=[\mathrm{B}]_{\mathrm{o}}-\left[\mathrm{BH}^{+}\right]_{\mathrm{o}}+x$

$\left[\mathrm{BHB}^{+}\right]=K_{\mathrm{f}}\left[\mathrm{BH}^{+}\right][\mathrm{B}]$

Substitute expressions for $\left[\mathrm{BHB}^{+}\right]$and $[\mathrm{B}]$, and collect terms:

$\left[\mathrm{BH}^{+}\right]_{\mathrm{o}}-\left[\mathrm{BH}^{+}\right]=K_{\mathrm{f}}\left[\mathrm{BH}^{+}\right]\left([\mathrm{B}]_{\mathrm{o}}-\left[\mathrm{BH}^{+}\right]_{\mathrm{o}}+\left[\mathrm{BH}^{+}\right]\right)$

$\left[\mathrm{BH}^{+}\right]_{\mathrm{o}}-\left[\mathrm{BH}^{+}\right]=K_{\mathrm{f}}\left[\mathrm{BH}^{+}\right][\mathrm{B}]_{\mathrm{o}}-K_{\mathrm{f}}\left[\mathrm{BH}^{+}\right]\left[\mathrm{BH}^{+}\right]_{\mathrm{o}}+K_{\mathrm{f}}\left[\mathrm{BH}^{+}\right]^{2}$

$K_{\mathrm{f}}\left[\mathrm{BH}^{+}\right]^{2}+\left\{K_{\mathrm{f}}\left([\mathrm{B}]_{\mathrm{o}}-\left[\mathrm{BH}^{+}\right]_{\mathrm{o}}\right)+1\right\}\left[\mathrm{BH}^{+}\right]-\left[\mathrm{BH}^{+}\right]_{\mathrm{o}}=0$

$\therefore\left[\mathrm{BH}^{+}\right]_{\mathrm{EQ}}=\frac{-\left\{K_{\mathrm{f}}\left([\mathrm{B}]_{\mathrm{o}}-\left[\mathrm{BH}^{+}\right]_{\mathrm{o}}\right)+1\right\}+\sqrt{\left\{K_{\mathrm{f}}\left([\mathrm{B}]_{\mathrm{o}}-\left[\mathrm{BH}^{+}\right]_{\mathrm{o}}\right)+1\right\}^{2}+4 K_{\mathrm{f}}\left[\mathrm{BH}^{+}\right]_{\mathrm{o}}}}{2 K_{\mathrm{f}}}$ 
Similarly, determine $[\mathrm{B}]_{\mathrm{EQ}}$ for initial concentrations $\left[\mathrm{BH}^{+}\right]_{\mathrm{o}}$ and $[\mathrm{B}]_{\mathrm{o}}$ :

$$
\begin{aligned}
& {[\mathrm{B}]=x ;\left[\mathrm{BHB}^{+}\right]=[\mathrm{B}]_{\mathrm{o}}-x ;\left[\mathrm{BH}^{+}\right]=\left[\mathrm{BH}^{+}\right]_{\mathrm{o}}-[\mathrm{B}]_{\mathrm{o}}+x} \\
& {[\mathrm{~B}]_{\mathrm{o}}-[\mathrm{B}]=K_{\mathrm{f}}[\mathrm{B}]\left(\left[\mathrm{BH}{ }^{+}\right]_{\mathrm{o}}-[\mathrm{B}]_{\mathrm{o}}+[\mathrm{B}]\right)} \\
& {[\mathrm{B}]_{\mathrm{o}}-[\mathrm{B}]=K_{\mathrm{f}}\left[\mathrm{BH}{ }^{+}\right]_{\mathrm{o}}[\mathrm{B}]-K_{\mathrm{f}}[\mathrm{B}]_{\mathrm{o}}[\mathrm{B}]+K_{\mathrm{f}}[\mathrm{B}]^{2}} \\
& K_{\mathrm{f}}[\mathrm{B}]^{2}+\left\{K_{\mathrm{f}}\left(\left[\mathrm{BH}^{+}\right]_{\mathrm{o}}-[\mathrm{B}]_{\mathrm{o}}\right)+1\right\}[\mathrm{B}]-[\mathrm{B}]_{\mathrm{o}}=0 \\
& \therefore[\mathrm{B}]_{\mathrm{EQ}}=\frac{-\left\{K_{\mathrm{f}}\left(\left[\mathrm{BH}^{+}\right]_{\mathrm{o}}-[\mathrm{B}]_{\mathrm{o}}\right)+1\right\}+\sqrt{\left\{K_{\mathrm{f}}\left(\left[\mathrm{BH}^{+}\right]_{\mathrm{o}}-[\mathrm{B}]_{\mathrm{o}}\right)+1\right\}^{2}+4 K_{\mathrm{f}}[\mathrm{B}]_{\mathrm{o}}}}{2 K_{\mathrm{f}}}
\end{aligned}
$$

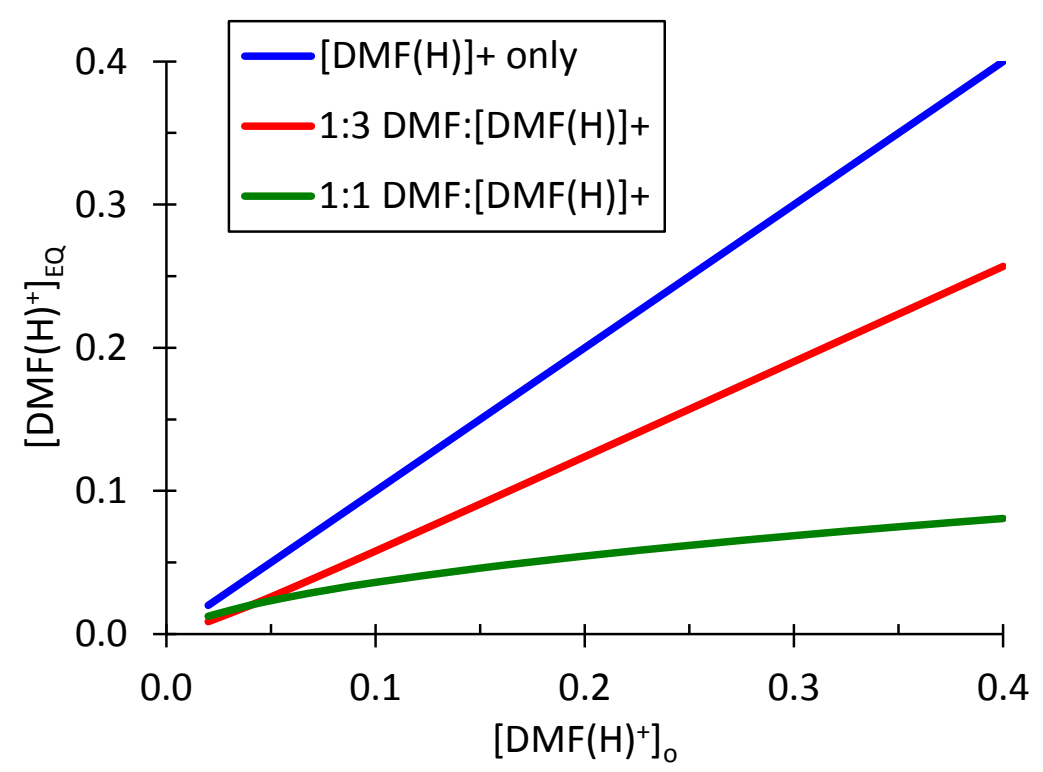

Figure S2. Plot of $\left[\mathrm{DMF}(\mathrm{H})^{+}\right]_{\mathrm{EQ}}$ vs $\left[\mathrm{DMF}(\mathrm{H})^{+}\right]_{\mathrm{o}}$ showing the effect of homoconjugation. 


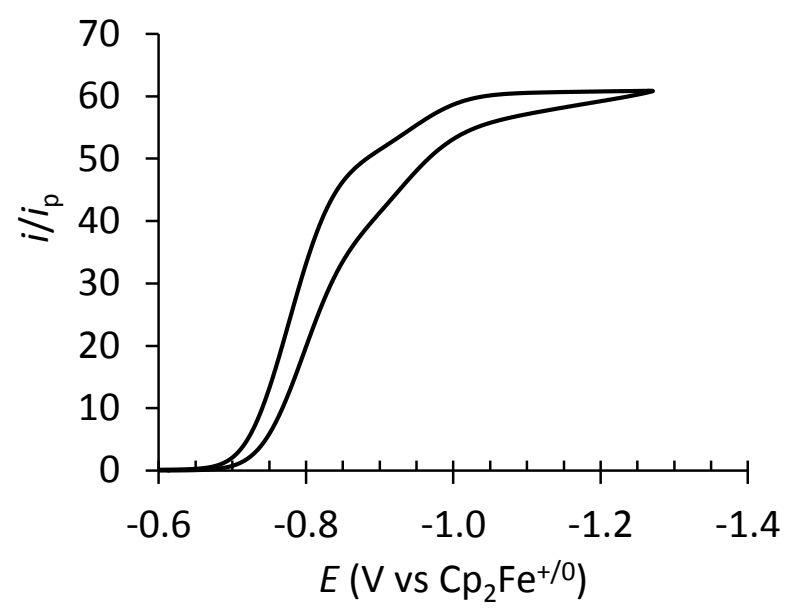

Figure S3. Cyclic voltammogram of 1 with 1:3 DMF:DMF $(\mathrm{H})^{+}$. Conditions: $1(0.7 \mathrm{mM}),[\mathrm{DMF}]_{\mathrm{o}}=55$ $\left.\mathrm{mM},\left[\mathrm{DMF}(\mathrm{H})^{+}\right]_{\mathrm{o}}=164 \mathrm{mM}\right),[\mathrm{DMF}]_{\mathrm{EQ}}=8 \mathrm{mM},\left[\mathrm{DMF}(\mathrm{H})^{+}\right]_{\mathrm{EQ}}=100 \mathrm{mM}, \mathrm{MeCN}\left(0.2 \mathrm{M} \mathrm{NBu}_{4} \mathrm{PF}_{6}\right), v$ $=0.05 \mathrm{~V} \mathrm{~s}^{-1}, 1 \mathrm{~mm}$ diam. glassy carbon working electrode.

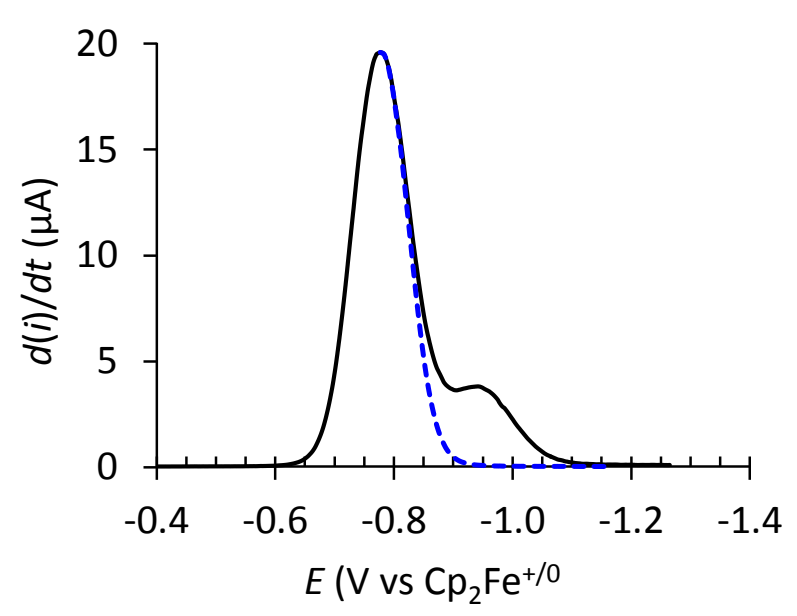

Figure S4. First-derivative trace of 1 with 1:3 $\operatorname{DMF}: \operatorname{DMF}(\mathrm{H})^{+}$, showing the reflection about the first maximum (dashed blue trace). Conditions: $\left.1(0.7 \mathrm{mM}),[D M F]_{0}=55 \mathrm{mM},\left[\mathrm{DMF}(\mathrm{H})^{+}\right]_{\mathrm{o}}=164 \mathrm{mM}\right)$, $[\mathrm{DMF}]_{\mathrm{EQ}}=8 \mathrm{mM},\left[\mathrm{DMF}(\mathrm{H})^{+}\right]_{\mathrm{EQ}}=100 \mathrm{mM}, \mathrm{MeCN}\left(0.2 \mathrm{M} \mathrm{NBu}_{4} \mathrm{PF}_{6}\right), v=0.05 \mathrm{~V} \mathrm{~s}^{-1}, 1 \mathrm{~mm}$ diam. glassy carbon working electrode. 


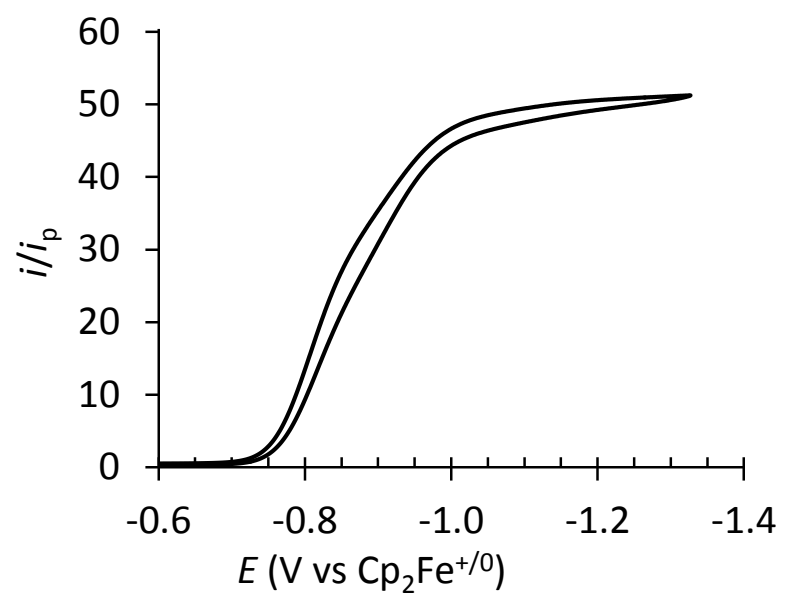

Figure S5. Cyclic voltammogram of 1 with 1:1 DMF:DMF $(\mathrm{H})^{+}$. Conditions: $1(0.8 \mathrm{mM}),[\mathrm{DMF}]_{\mathrm{o}}=$ $\left[\mathrm{DMF}(\mathrm{H})^{+}\right]_{\mathrm{o}}=129 \mathrm{mM},[\mathrm{DMF}]_{\mathrm{EQ}}=\left[\mathrm{DMF}(\mathrm{H})^{+}\right]_{\mathrm{EQ}}=42 \mathrm{mM}, \mathrm{MeCN}\left(0.2 \mathrm{M} \mathrm{NBu}_{4} \mathrm{PF}_{6}\right), v=0.05 \mathrm{~V} \mathrm{~s}^{-1}$, $1 \mathrm{~mm}$ diam. glassy carbon working electrode.

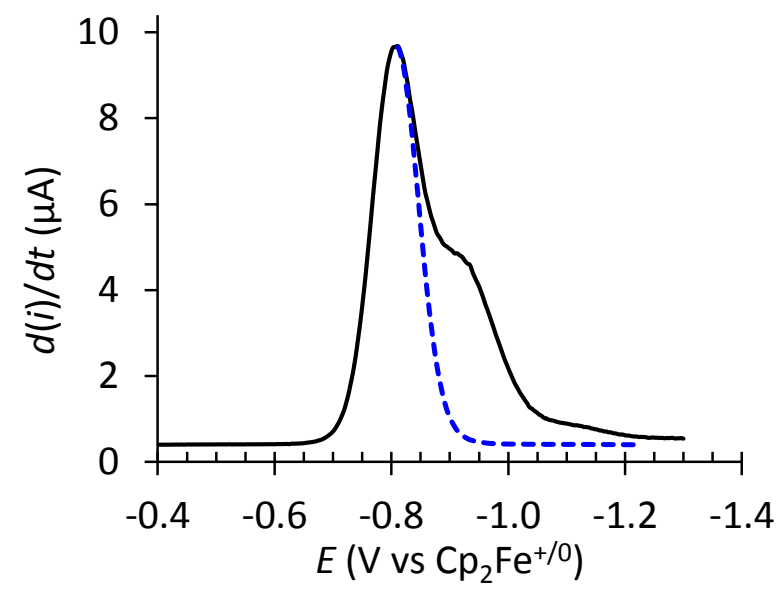

Figure S6. First-derivative trace of 1 with 1:1 DMF:DMF(H) ${ }^{+}$, showing the reflection about the first maximum (dashed blue trace). Conditions: $1(0.8 \mathrm{mM}),[\mathrm{DMF}]_{\mathrm{o}}=\left[\mathrm{DMF}(\mathrm{H})^{+}\right]_{\mathrm{o}}(129 \mathrm{mM}),[\mathrm{DMF}]_{\mathrm{EQ}}=$ $\left[\mathrm{DMF}(\mathrm{H})^{+}\right]_{\mathrm{EQ}}(42 \mathrm{mM}), \mathrm{MeCN}\left(0.2 \mathrm{M} \mathrm{NBu}_{4} \mathrm{PF}_{6}\right), v=0.05 \mathrm{~V} \mathrm{~s}^{-1}, 1 \mathrm{~mm}$ diam. glassy carbon working electrode. 
Table S1. Tabulation of kinetic data for ECEC mechanism of $\mathbf{1}$ with unbuffered $\operatorname{DMF}(\mathrm{H})^{+}$.

\begin{tabular}{|l|l|l|l|l|l|l|l|l|}
\hline $\begin{array}{l}{\left[\mathrm{DMF}(\mathrm{H})^{+}\right]_{\mathrm{o}}} \\
(\mathrm{M})\end{array}$ & $\begin{array}{l}E_{\text {cat } / 2}{ }^{a} \\
(\mathrm{~V})\end{array}$ & $\lambda$ & $\begin{array}{l}i_{\mathrm{p}}{ }^{b} \\
(\mu \mathrm{A})\end{array}$ & $\begin{array}{l}i \text { at } E_{\text {cat } / 2} \\
(\mu \mathrm{A})\end{array}$ & $i_{\text {cat }} / i_{\mathrm{p}}$ & $\begin{array}{l}k_{\text {obs }} \\
\left(\mathrm{s}^{-1}\right)\end{array}$ & $\begin{array}{l}k_{1} \\
\left(\mathrm{~s}^{-1}\right)\end{array}$ & $\begin{array}{l}k_{2} \\
\left(\mathrm{~s}^{-1}\right)\end{array}$ \\
\hline 0.007 & -0.784 & 5.0 & 1.23 & 2.31 & 3.75 & 5 & 196 & 8 \\
\hline 0.015 & -0.777 & 6.9 & 1.22 & 3.70 & 6.06 & 14 & 882 & 19 \\
\hline 0.021 & -0.776 & 7.2 & 1.20 & 4.84 & 8.06 & 25 & 1687 & 33 \\
\hline 0.028 & -0.775 & 7.5 & 1.18 & 5.57 & 9.44 & 35 & 2501 & 44 \\
\hline 0.040 & -0.775 & 7.5 & 1.18 & 7.32 & 12.4 & 59 & 4301 & 76 \\
\hline 0.053 & -0.774 & 7.8 & 1.11 & 8.27 & 14.9 & 86 & 6735 & 110 \\
\hline 0.064 & -0.769 & 9.7 & 1.07 & 9.84 & 18.4 & 131 & 15091 & 159 \\
\hline 0.076 & -0.773 & 8.2 & 1.06 & 10.8 & 20.3 & 160 & 13499 & 201 \\
\hline 0.086 & -0.767 & 10.6 & 1.03 & 11.1 & 21.6 & 181 & 24383 & 217 \\
\hline 0.106 & -0.770 & 9.3 & 0.99 & 11.2 & 22.6 & 197 & 21060 & 242 \\
\hline 0.125 & -0.769 & 9.7 & 0.95 & 11.8 & 24.9 & 240 & 27712 & 292 \\
\hline 0.158 & -0.763 & 12.6 & 0.88 & 11.6 & 26.3 & 268 & 49286 & 312 \\
\hline 0.185 & -0.762 & 13.1 & 0.82 & 10.7 & 26.2 & 266 & 52992 & 309 \\
\hline 0.210 & -0.760 & 14.2 & 0.77 & 10.2 & 26.6 & 274 & 63775 & 314 \\
\hline 0.235 & -0.758 & 15.5 & 0.73 & 9.78 & 26.8 & 278 & 75418 & 315 \\
\hline
\end{tabular}

${ }^{a}$ Measured from the first maximum of the first-derivative trace. ${ }^{b}$ Adjusted for dilution by comparison to the internal $\mathrm{Cp}_{2} \mathrm{Fe}^{+/ 0}$ reference.

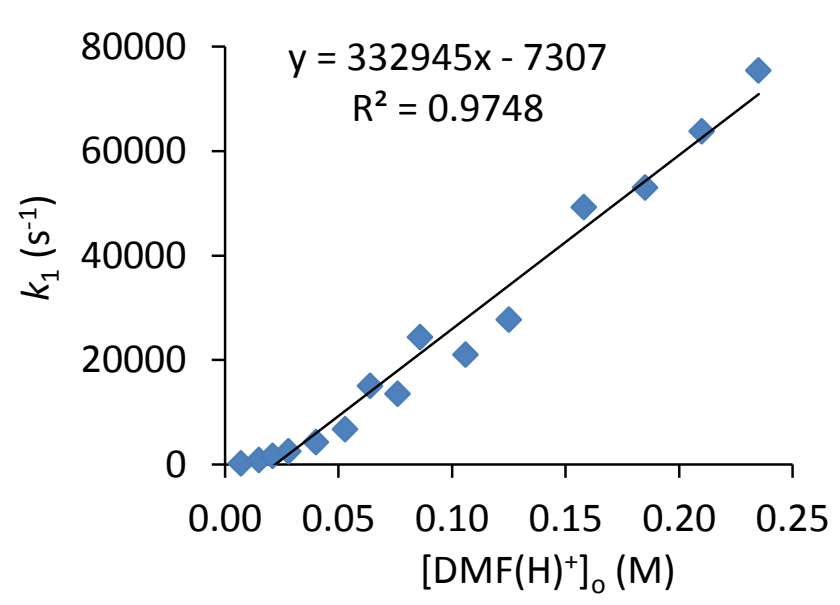

Figure S7. Plot of $k_{1}$ for ECEC measured from $E_{\text {cat } / 2}$ analysis versus $\left[\mathrm{DMF}(\mathrm{H})^{+}\right]_{\mathrm{o}}$ using unbuffered $\operatorname{DMF}(\mathrm{H})^{+}$. 
Table S2. Tabulation of kinetic data for ECEC mechanism of 1 with 1:3 DMF:DMF(H) ${ }^{+}$.

\begin{tabular}{|l|l|l|l|l|l|l|l|l|l|}
\hline $\begin{array}{l}{\left[\mathrm{DMF}(\mathrm{H})^{+}\right]_{\mathrm{o}}{ }^{a}} \\
(\mathrm{M})\end{array}$ & $\begin{array}{l}{\left[\mathrm{DMF}(\mathrm{H})^{+}\right]_{\mathrm{EQ}}} \\
(\mathrm{M})\end{array}$ & $\begin{array}{l}E_{\text {cat/2 }}{ }^{b} \\
(\mathrm{~V})\end{array}$ & $\lambda$ & $\begin{array}{l}i_{\mathrm{p}}{ }^{c} \\
(\mu \mathrm{A})\end{array}$ & $\begin{array}{l}i \text { at } E_{\text {cat } / 2} \\
(\mu \mathrm{A})\end{array}$ & $i_{\text {cat }} / i_{\mathrm{p}}$ & $\begin{array}{l}k_{\text {obs }} \\
\left(\mathrm{s}^{-1}\right)\end{array}$ & $\begin{array}{l}k_{1} \\
\left(\mathrm{~s}^{-1}\right)\end{array}$ & $\begin{array}{l}k_{2} \\
\left(\mathrm{~s}^{-1}\right)\end{array}$ \\
\hline 0.008 & 0.003 & -0.792 & 3.39 & 1.29 & 1.94 & 3.00 & 3 & 67 & 6 \\
\hline 0.015 & 0.006 & -0.788 & 4.13 & 1.28 & 3.49 & 5.46 & 12 & 304 & 18 \\
\hline 0.022 & 0.010 & -0.786 & 4.54 & 1.26 & 4.61 & 7.31 & 21 & 637 & 31 \\
\hline 0.029 & 0.013 & -0.786 & 4.54 & 1.24 & 5.62 & 9.06 & 32 & 978 & 47 \\
\hline 0.042 & 0.021 & -0.783 & 5.23 & 1.21 & 6.90 & 11.4 & 51 & 1960 & 72 \\
\hline 0.055 & 0.029 & -0.780 & 6.00 & 1.19 & 7.62 & 12.8 & 64 & 3130 & 87 \\
\hline 0.067 & 0.037 & -0.783 & 5.23 & 1.15 & 8.28 & 14.4 & 80 & 3105 & 114 \\
\hline 0.079 & 0.044 & -0.780 & 6.00 & 1.13 & 9.04 & 16.0 & 99 & 4844 & 135 \\
\hline 0.090 & 0.051 & -0.778 & 6.57 & 1.10 & 9.24 & 16.8 & 110 & 6301 & 146 \\
\hline 0.110 & 0.065 & -0.780 & 6.00 & 1.05 & 10.2 & 19.4 & 145 & 7127 & 198 \\
\hline 0.130 & 0.077 & -0.779 & 6.28 & 1.01 & 10.4 & 20.5 & 163 & 8638 & 219 \\
\hline 0.164 & 0.100 & -0.777 & 6.87 & 0.94 & 11.2 & 23.9 & 221 & 13696 & 290 \\
\hline
\end{tabular}

${ }^{a}[\mathrm{DMF}]_{\mathrm{o}}=0.33 \times\left[\mathrm{DMF}(\mathrm{H})^{+}\right]_{\mathrm{o}}{ }^{b}$ Measured from the first maximum of the first-derivative trace. ${ }^{c}$ Adjusted for dilution by comparison to the internal $\mathrm{Cp}_{2} \mathrm{Fe}^{+/ 0}$ reference.

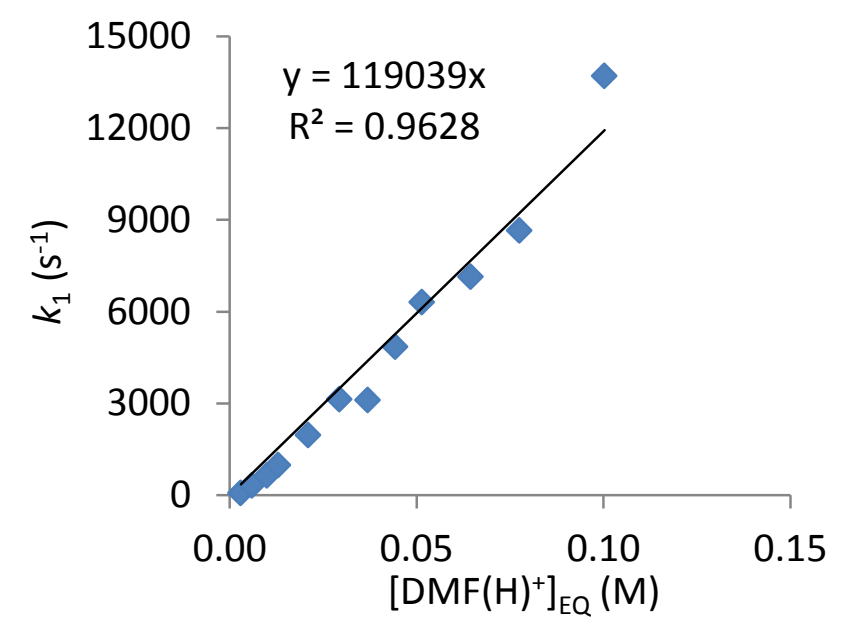

Figure S8. Plot of $k_{1}$ for ECEC measured from $E_{\text {cat } / 2}$ analysis versus $\left[\mathrm{DMF}(\mathrm{H})^{+}\right]_{\mathrm{EQ}}$ using 1:3 $\operatorname{DMF}: \operatorname{DMF}(\mathrm{H})^{+}$. 
Table S3. Tabulation of kinetic data for ECEC mechanism of 1 with 1:1 DMF:DMF(H) ${ }^{+}$.

\begin{tabular}{|l|l|l|l|l|l|l|l|l|l|}
\hline $\begin{array}{l}{\left[\mathrm{DMF}(\mathrm{H})^{+}\right]_{\mathrm{o}}{ }^{a}} \\
(\mathrm{M})\end{array}$ & $\begin{array}{l}{\left[\mathrm{DMF}(\mathrm{H})^{+}\right]_{\mathrm{EQ}}} \\
(\mathrm{M})\end{array}$ & $\begin{array}{l}E_{\mathrm{cat} / 2}{ }^{b} \\
(\mathrm{~V})\end{array}$ & $\lambda$ & $\begin{array}{l}i_{\mathrm{p}}{ }^{c} \\
(\mu \mathrm{A})\end{array}$ & $\begin{array}{l}i \text { at } E_{\text {cat } / 2} \\
(\mu \mathrm{A})\end{array}$ & $i_{\text {cat }} / i_{\mathrm{p}}$ & $\begin{array}{l}k_{\text {obs }} \\
\left(\mathrm{s}^{-1}\right)\end{array}$ & $\begin{array}{l}k_{1} \\
\left(\mathrm{~s}^{-1}\right)\end{array}$ & $\begin{array}{l}k_{2} \\
\left(\mathrm{~s}^{-1}\right)\end{array}$ \\
\hline 0.007 & 0.006 & -0.803 & 1.86 & 0.779 & 1.63 & 4.18 & 7 & 55 & 16 \\
\hline 0.014 & 0.010 & -0.801 & 2.09 & 0.764 & 2.31 & 6.06 & 14 & 136 & 31 \\
\hline 0.022 & 0.013 & -0.803 & 1.86 & 0.760 & 2.79 & 7.35 & 21 & 171 & 50 \\
\hline 0.028 & 0.016 & -0.801 & 2.09 & 0.751 & 3.20 & 8.52 & 28 & 269 & 62 \\
\hline 0.041 & 0.021 & -0.803 & 1.86 & 0.726 & 3.55 & 9.78 & 37 & 304 & 88 \\
\hline 0.055 & 0.025 & -0.807 & 1.45 & 0.719 & 4.06 & 11.3 & 50 & 297 & 142 \\
\hline 0.066 & 0.028 & -0.808 & 1.35 & 0.696 & 4.04 & 11.6 & 52 & 289 & 158 \\
\hline 0.078 & 0.031 & -0.805 & 1.65 & 0.686 & 4.46 & 13.0 & 66 & 462 & 171 \\
\hline 0.089 & 0.034 & -0.808 & 1.35 & 0.667 & 4.57 & 13.7 & 73 & 402 & 219 \\
\hline 0.109 & 0.038 & -0.804 & 1.75 & 0.640 & 4.70 & 14.7 & 84 & 636 & 208 \\
\hline 0.129 & 0.042 & -0.807 & 1.45 & 0.618 & 4.70 & 15.2 & 89 & 534 & 255 \\
\hline
\end{tabular}

${ }^{a}[\mathrm{DMF}]_{\mathrm{o}}=\left[\mathrm{DMF}(\mathrm{H})^{+}\right]_{\mathrm{o}}{ }^{b}$ Measured from the first maximum of the first-derivative trace. ${ }^{c}$ Adjusted for dilution by comparison to the internal $\mathrm{Cp}_{2} \mathrm{Fe}^{+/ 0}$ reference.

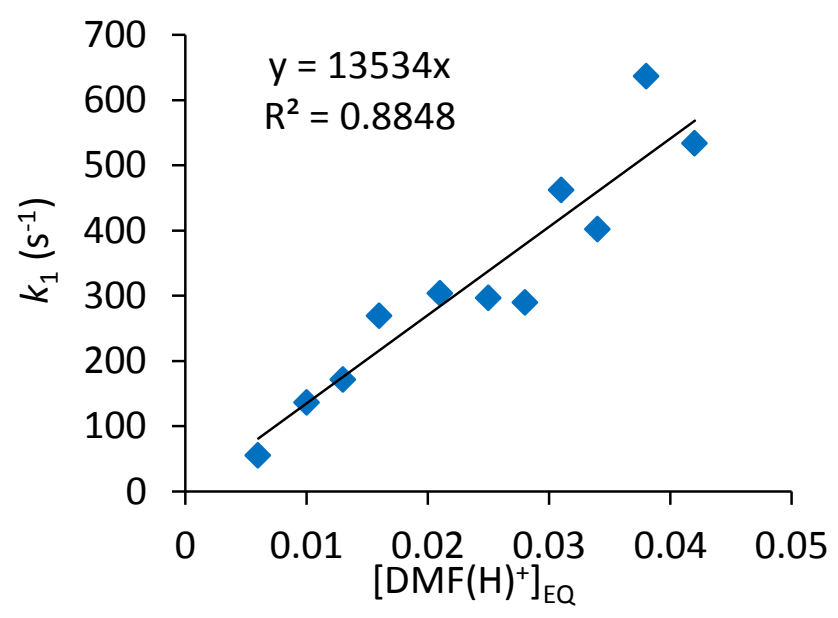

Figure S9. Plot of $k_{1}$ for ECEC measured from $E_{\text {cat } / 2}$ analysis versus [DMF $\left.(\mathrm{H})^{+}\right]_{\mathrm{EQ}}$ using $1: 1$ DMF:DMF $(\mathrm{H})^{+}$. 
Estimate of $\boldsymbol{k}_{\mathbf{1}}$ and $\boldsymbol{k}_{\mathbf{2}}$ for EECC Pathway. As described in the main text, FOWA cannot be used to determine $k_{1}$ for the EECC pathway of $\mathbf{1}$ due to the preceding ECEC mechanism. Additionally, the second maximum in the first-derivative plot corresponds to $50 \%$ of 1 operating via ECEC and $50 \%$ via EECC, which is not the same as $E_{\text {cat } / 2}$ for the EECC pathway. However, since the true $E_{\text {cat } / 2}$ should more positive than the second maximum (see Fig $6 \mathrm{~b}$ in the main text), then the second maximum represents a lower limit of $E_{\text {cat } / 2}$, and analysis according to the EECC equations in Table 1 can provide a lower limit on $k_{1}$, shown in Figure S10. Additionally, the lower limit estimates of $k_{1}$ can be used to determine an upper limit estimate of $k_{2}$. The upper limit estimates of $k_{2}$ obtained in this manner are identical to the corresponding $k_{\mathrm{obs}}$ values, shown in Figure S11.

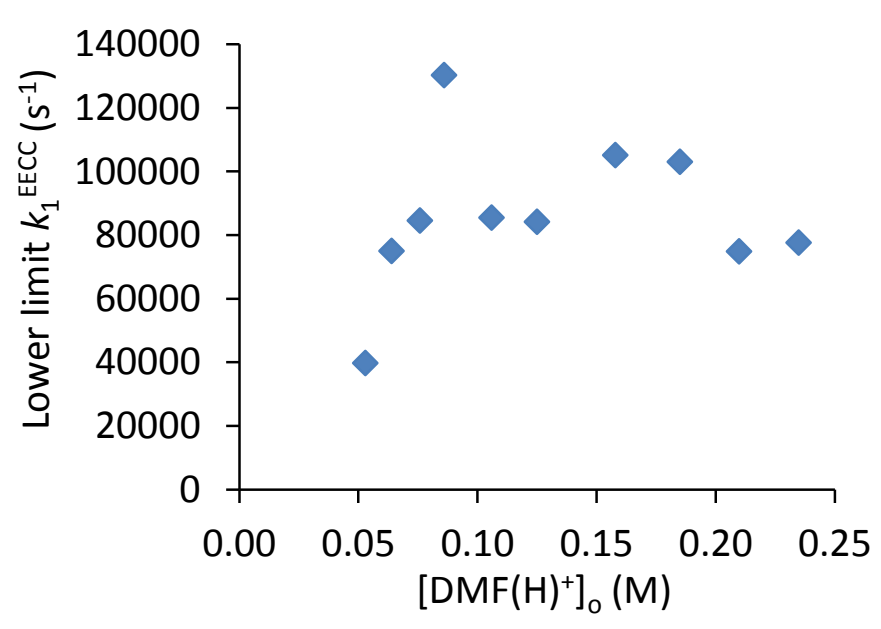

Figure S10. Plot of the lower limit on $k_{1}$ for EECC versus $\left[\mathrm{DMF}(\mathrm{H})^{+}\right]_{\mathrm{o}}$ using unbuffered $\mathrm{DMF}(\mathrm{H})^{+}$, measured from $E_{\mathrm{cat} / 2}$ analysis using the second maximum in the first derivative plot as an estimate for $E_{\text {cat } / 2 \text {. }}$

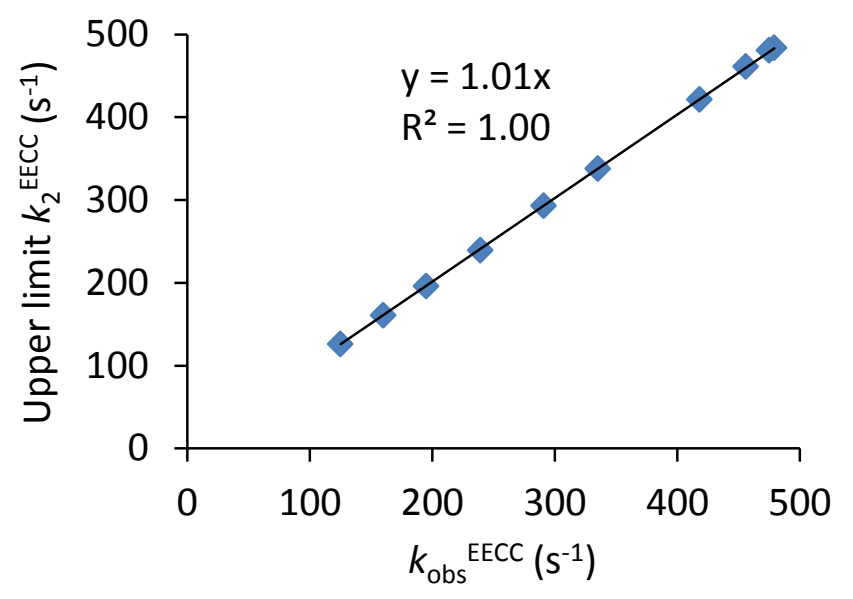

Figure S11. Plot of the upper limit on $k_{2}$ for EECC versus $k_{\text {obs }}$ using unbuffered $\operatorname{DMF}(\mathrm{H})^{+}$. 


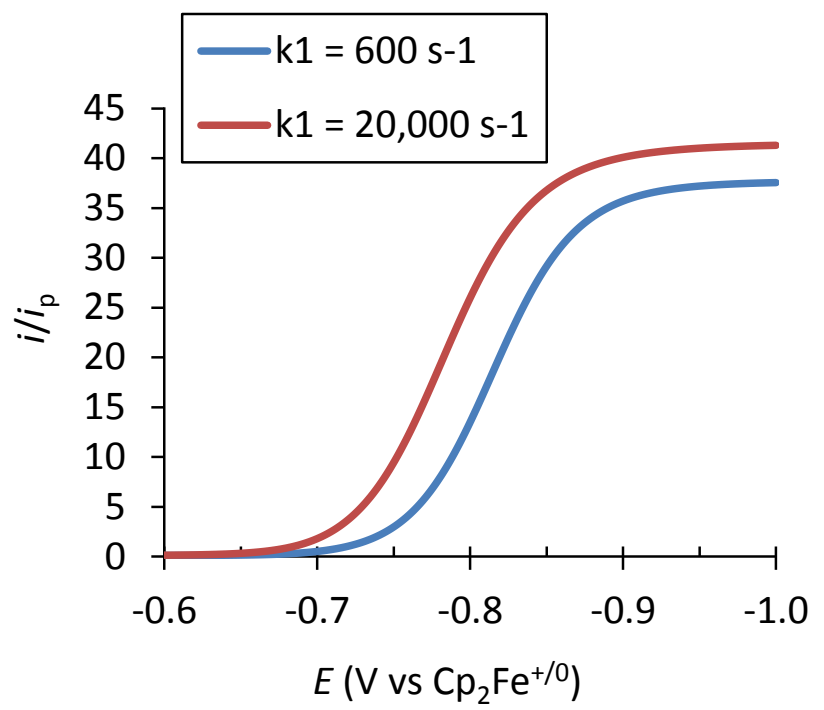

Figure S12. Simulated voltammograms for an ECEC mechanism with $E_{2}$ more negative than the foot of the wave. ECEC Model: $E_{1}=-0.83 \mathrm{~V}, E_{2}=-0.75 \mathrm{~V}, k_{1}=$ variable, $k_{2}=500 \mathrm{~s}^{-1}, v=0.05 \mathrm{~V} \mathrm{~s}^{-1}, D=$ $1 \times 10^{-5} \mathrm{~cm}^{2} \mathrm{~s}^{-1}, k_{\mathrm{s}}=0.5 \mathrm{~cm} \mathrm{~s}^{-1}$.

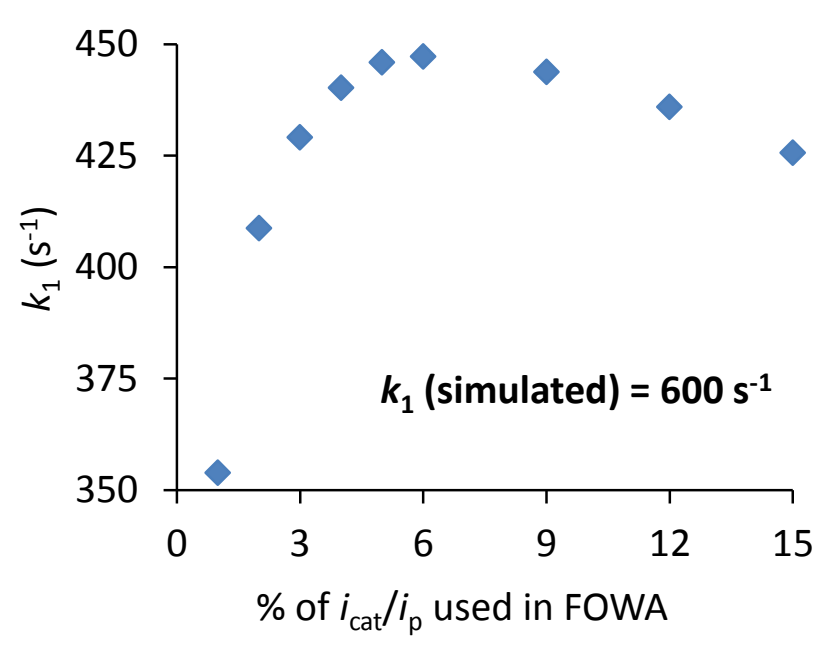

Figure S13. Plot of $k_{1}$ versus the percent of $i_{\text {cat }} / i_{\mathrm{p}}$ used in FOWA for an ECEC mechanism with $E_{2}$ more negative than the foot of the wave. ECEC Model: $E_{1}=-0.83 \mathrm{~V}, E_{2}=-0.75 \mathrm{~V}, k_{1}=600 \mathrm{~s}^{-1}, k_{2}=500 \mathrm{~s}^{-1}$, $v=0.05 \mathrm{~V} \mathrm{~s}^{-1}, D=1 \times 10^{-5} \mathrm{~cm}^{2} \mathrm{~s}^{-1}, k_{\mathrm{s}}=0.5 \mathrm{~cm} \mathrm{~s}^{-1}$. 


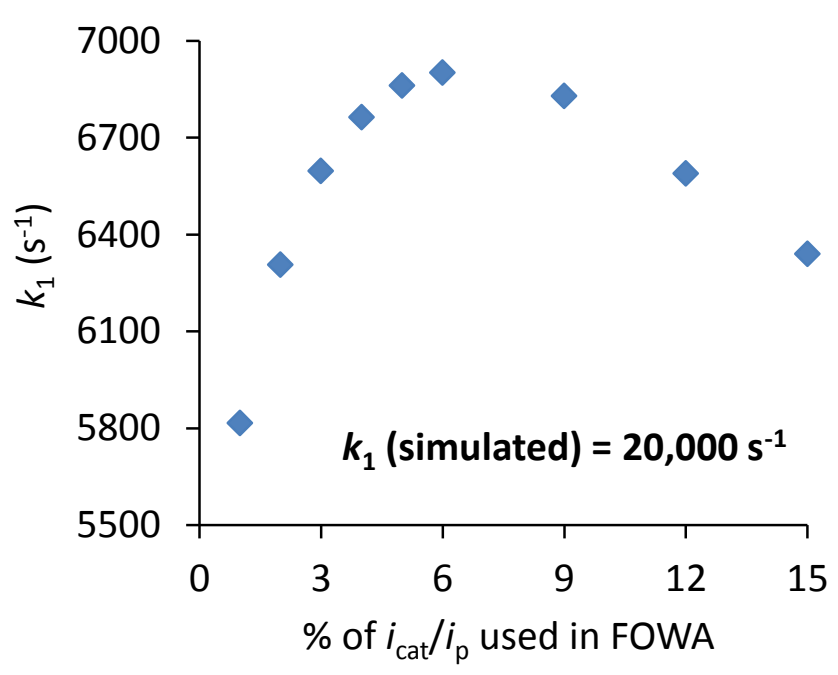

Figure S14. Plot of $k_{1}$ versus the percent of $i_{\text {cat }} / i_{\mathrm{p}}$ used in FOWA for an ECEC mechanism with $E_{2}$ more negative than the foot of the wave. ECEC Model: $E_{1}=-0.83 \mathrm{~V}, E_{2}=-0.75 \mathrm{~V}, k_{1}=20,000 \mathrm{~s}^{-1}, k_{2}=500$ $\mathrm{s}^{-1}, v=0.05 \mathrm{~V} \mathrm{~s}^{-1}, D=1 \times 10^{-5} \mathrm{~cm}^{2} \mathrm{~s}^{-1}, k_{\mathrm{s}}=0.5 \mathrm{~cm} \mathrm{~s}^{-1}$.

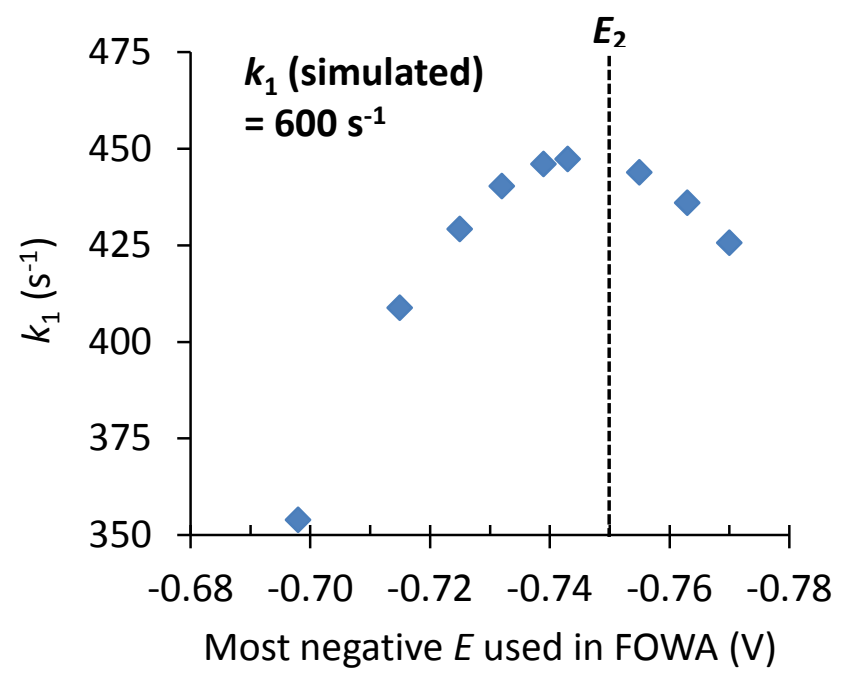

Figure S15. Plot of $k_{1}$ versus the most negative potential used in FOWA for an ECEC mechanism with $E_{2}$ more negative than the foot of the wave. ECEC Model: $E_{1}=-0.83 \mathrm{~V}, E_{2}=-0.75 \mathrm{~V}, k_{1}=600 \mathrm{~s}^{-1}, k_{2}=$ $500 \mathrm{~s}^{-1}, v=0.05 \mathrm{~V} \mathrm{~s}^{-1}, D=1 \times 10^{-5} \mathrm{~cm}^{2} \mathrm{~s}^{-1}, k_{\mathrm{s}}=0.5 \mathrm{~cm} \mathrm{~s}^{-1}$. 


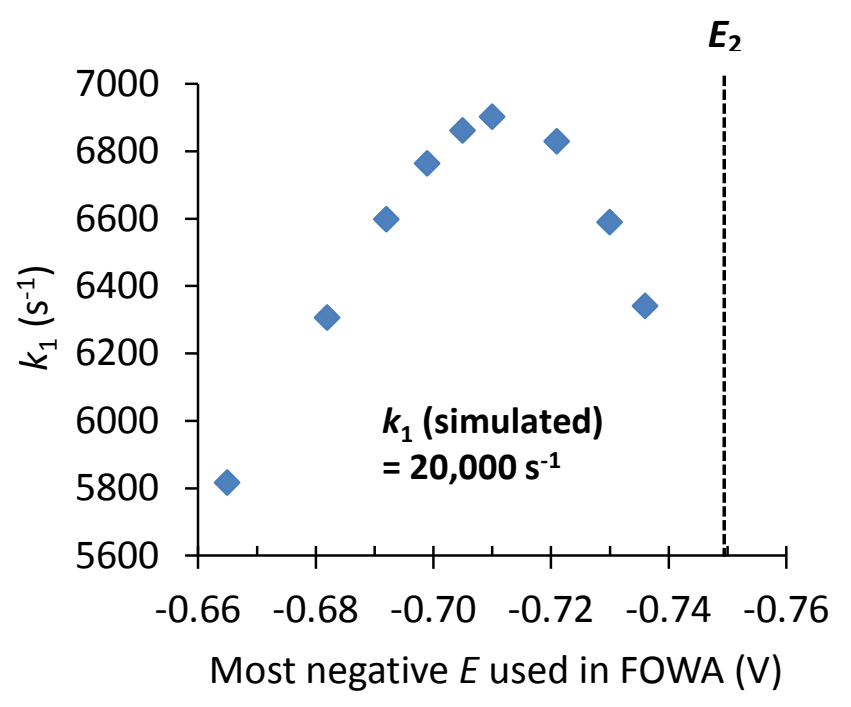

Figure S16. Plot of $k_{1}$ versus the most negative potential used in FOWA for an ECEC mechanism with $E_{2}$ more negative than the foot of the wave. ECEC Model: $E_{1}=-0.83 \mathrm{~V}, E_{2}=-0.75 \mathrm{~V}, k_{1}=20,000 \mathrm{~s}^{-1}$, $k_{2}=500 \mathrm{~s}^{-1}, v=0.05 \mathrm{~V} \mathrm{~s}^{-1}, D=1 \times 10^{-5} \mathrm{~cm}^{2} \mathrm{~s}^{-1}, k_{\mathrm{s}}=0.5 \mathrm{~cm} \mathrm{~s}^{-1}$.

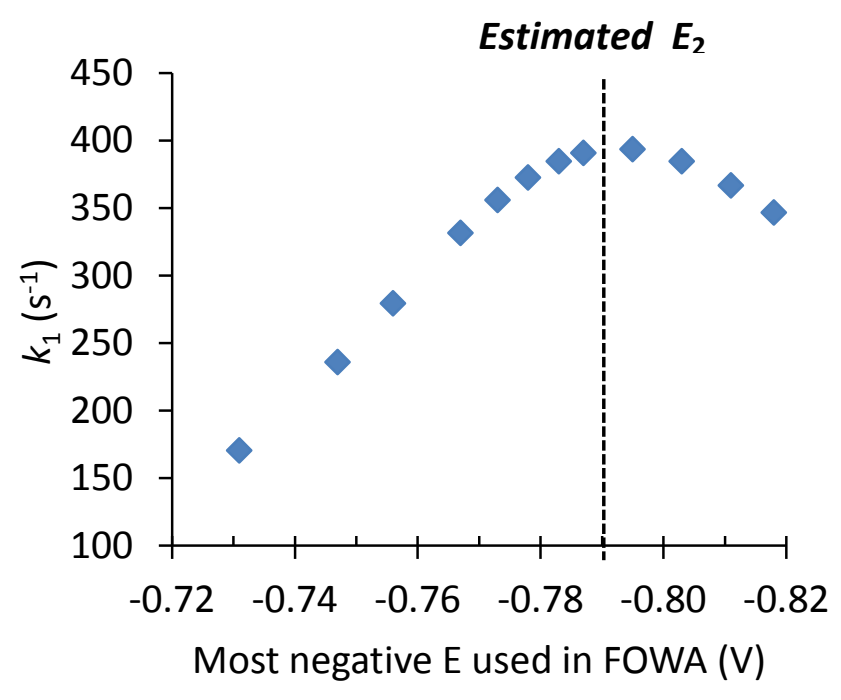

Figure S17. Plot of $k_{1}$ versus the most negative potential used in FOWA for 1 with 1:1 DMF:DMF(H) ${ }^{+}$. Conditions: $1(0.8 \mathrm{mM}),[\mathrm{DMF}]_{\mathrm{o}}=\left[\mathrm{DMF}(\mathrm{H})^{+}\right]_{\mathrm{o}}=217 \mathrm{mM},[\mathrm{DMF}]_{\mathrm{EQ}}=\left[\mathrm{DMF}(\mathrm{H})^{+}\right]_{\mathrm{EQ}}=57 \mathrm{mM}, \mathrm{MeCN}$ $\left(0.2 \mathrm{M} \mathrm{NBu}_{4} \mathrm{PF}_{6}\right), v=0.05 \mathrm{~V} \mathrm{~s}^{-1}, 1 \mathrm{~mm}$ diam. glassy carbon working electrode. 


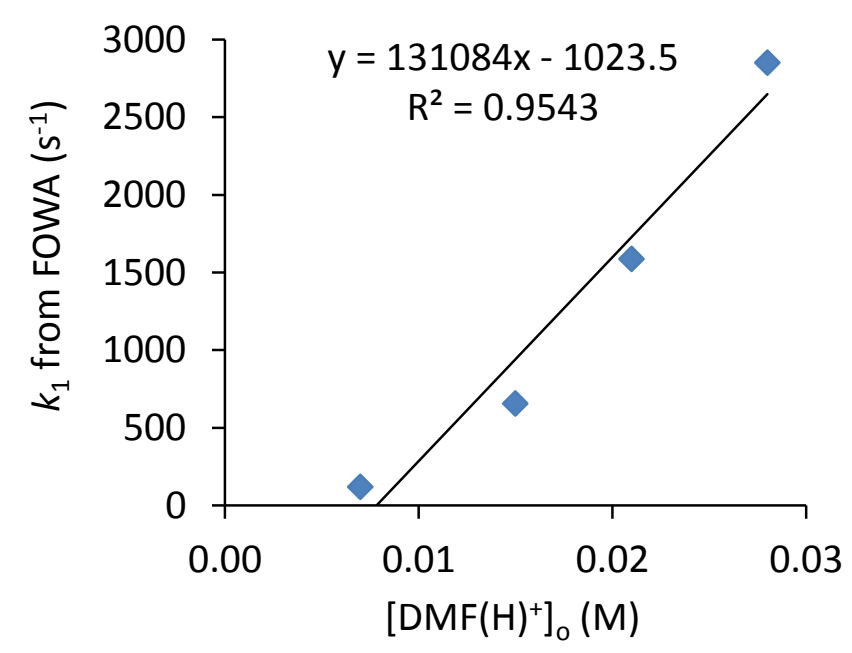

Figure S18. Plot of $k_{1}$ for ECEC measured from FOWA $\left(3 \% i_{\text {cat }} / i_{\mathrm{p}}\right)$ versus $\left[\mathrm{DMF}(\mathrm{H})^{+}\right]_{\mathrm{o}}$ using unbuffered $\mathrm{DMF}(\mathrm{H})^{+}$. The measured $k_{1}$ is first order in acid at low concentrations of acid.

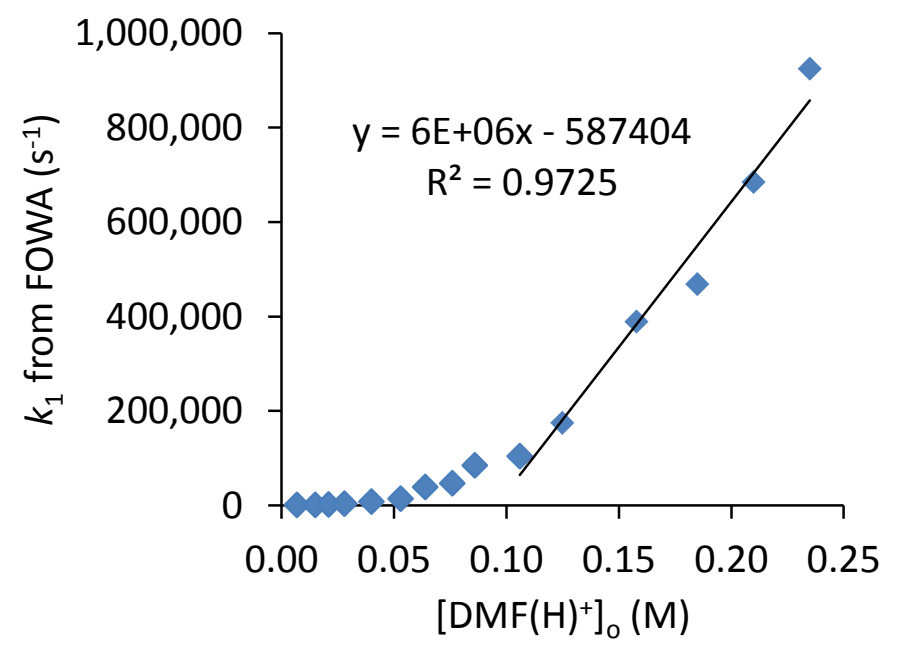

Figure S19. Plot of $k_{1}$ for ECEC measured from FOWA $\left(3 \% i_{\text {cat }} / i_{\mathrm{p}}\right)$ versus $\left[\mathrm{DMF}(\mathrm{H})^{+}\right]_{\mathrm{o}}$ using unbuffered $\operatorname{DMF}(\mathrm{H})^{+}$. A change in slope is observed at high concentrations of acid, suggesting a change in mechanism. 